\title{
A THEORY OF UNBOUNDED GENERALIZED SCALAR OPERATORS
}

\author{
BRIAN KRITT
}

\begin{abstract}
ABSTRACr. In this paper is presented a theory of unbounded operators which admit spectral distributions. The compactness of the support in the bounded case has been replaced by certain growth conditions at infinity. Properties of these operators are studied, including the existence of a Spectral Mapping Theorem.
\end{abstract}

Introduction. In [2], Foias introduced the class of bounded operators in Banach spaces which in lieu of spectral measures admit spectral distributions. He proved certain basic properties of these "generalized scalars", such as the Spectral Mapping Thorem. Further investigations of this class of operators and of spectral distributions were made by various authors in $[1],[2],[4],[5],[6],{ }^{1}[8]$, and [10].

In [5], the present author studied the class of generalized scalars with real spectrum, which were called generalized pseudo-hermitian (g.p.h) operators. These operators were characterized by the elementary condition that the real exponential group generated by such an operator have polynomial growth in the uniform operator norm. ${ }^{2}$ In attempting to study the spectral properties of the infinitesimal generators of $C_{0}$-groups with polynomial growth, the author has been led to consider unbounded generalized scalar operators.

In this paper is presented a theory of unbounded spectral distributions and of the unbounded generalized scalar operators which they represent. The compactness of the support in the bounded case has been replaced by certain growth conditions at infinity sufficient to insure that the definitions are correct and the resulting class of operators well behaved, in that they generalize both the bounded generalized scalars and the unbounded normal operators in Hilbert space. Some properties of the class are discussed, culminating in a Spectral Mapping Theorem.

1. Basic definitions.

Definition 1. Let $T$ be a mapping from the space $\mathscr{D}\left(R^{2}\right)$ of complex test functions into the space $\mathscr{L}(X)$ of linear continuous operators on a

Received by the editors April 6, 1971.

4MS 1970 subject classifications. Primary 47A65, 47A60, 47B40; Secondary 46F99.

1 Also considers unbounded operators.

${ }^{2} \mathrm{Cf}$. Theorem 2 of [10].

(c) American Mathematical Society 1972 
Banach space $X$. (a) We say $T$ strongly vanishes to power $p$ at infinity for a vector $x \in X$ if and only if $T_{\lambda^{p} \varphi} x \rightarrow 0$ as $\varphi \in \mathscr{D}\left(R^{2}\right)$ varies through a family of test functions in such a manner that $\left\|T_{\varphi}\right\|$ remains bounded, and $\inf \{|\lambda|: \lambda \in$ support $\varphi\} \rightarrow \infty$.

(b) If $T$ strongly vanishes to power $p$ at infinity for all $x \in X$, we say $T$ strongly vanishes to power $p$ at infinity.

EXAMPLE. Let $E$ be a spectral measure in Hilbert space $H$, defined on the Borel sets in the complex plane, and define a mapping $T$ from $\mathscr{D}\left(R^{2}\right)$ into $\mathscr{L}(H)$ by the formula

$$
T_{\varphi}=\int \varphi d E \quad \text { for } \varphi \in \mathscr{D}\left(R^{2}\right)
$$

Here the integral is as defined in [4]. (Reference [7] provides some results used in the manipulations that follow.) Then we have (a) $T$ strongly vanishes to power 0 at infinity; and (b) $T$ strongly vanishes to power 1 at infinity for all $x$ in the domain $D_{A}$ of the operator $A$ given by the formula

$$
A=\int \lambda d E
$$

We remark that equation (2) is the general form for an unbounded normal operator in $H$.

PROOF OF (a).

$$
\left\|T_{\varphi} x\right\|^{2}=\int|\varphi|^{2} d \mu_{x}
$$

where $\mu_{x}$ is the measure $(E(\cdot) x, x)$. If $\varphi$ is a test function with support disjoint from the ball $S_{r}$ of radius $r$ about the origin in $R^{2}$, then

$$
\begin{aligned}
\left\|T_{\varphi} x\right\|^{2} & =\int_{R^{2} \mid S_{r}}|\varphi|^{2} d \mu_{x}=\left(\int|\varphi|^{2} d E x, E\left(R^{2} \mid S_{r}\right) x\right) \\
& \leqq\left\|E\left(R^{2} \mid S_{r}\right) x\right\| \cdot\left\|\int|\varphi|^{2} d E x\right\| \\
& =\mu_{x}\left(R^{2} \mid S_{r}\right) \cdot\left(\int|\varphi|^{4} d \mu_{x}\right)^{1 / 2} .
\end{aligned}
$$

Now $\int|\varphi|^{4} d \mu_{x}=\left\|T_{\varphi^{2}} x\right\|^{2}=\left\|T_{\varphi}^{2} x\right\|^{2} \leqq\left\|T_{\varphi}\right\|^{4}\|x\|^{2}$, and is hence bounded on a set of $\varphi$ for which $\left\|T_{\varphi}\right\|$ is bounded. Thus (4) implies that $T$ strongly vanishes to power 0 at infinity.

Proof of (b). If $x \in D_{A}$, then $\int|\lambda|^{2} d \mu_{x}<\infty$, so for supp $\varphi \cap S_{r}=\varnothing$, 
we have

$$
\begin{aligned}
\left\|T_{\lambda \varphi} x\right\|^{2} & =\int_{R^{2} \mid S_{r}}|\lambda|^{2}|\varphi|^{2} d \mu_{x} \\
& =\left(\int_{R^{2} \mid S_{r}}|\lambda| d E x, \quad \int|\lambda||\varphi|^{2} d E x\right) \\
& \leqq\left(\int_{R^{2} \mid S_{r}}|\lambda|^{2} d \mu_{x}\right)^{1 / 2} \cdot\left\|\int|\lambda||\varphi|^{2} d E x\right\| \\
& =\left(\int_{R^{2} \mid S_{r}}|\lambda|^{2} d \mu_{x}\right)^{1 / 2} \cdot\left\|\int|\varphi|^{2} d E y\right\|,
\end{aligned}
$$

where $y=\int|\lambda| d E x$. The second factor on the right side of (5) is bounded on a set of $\varphi$ for which $\left\|T_{\varphi}\right\|$ is bounded, as above. Hence the fact that $T$ strongly vanishes to power 1 at infinity for $x$ follows from the relation $\int|\lambda|^{2} d \mu_{x}<\infty$.

DEFINITION 2. Let $T$ be an "integrable" distribution of operators in $R^{2}$; i.e., $T$ is a linear mapping from $\mathscr{D}\left(R^{2}\right)$ into $\mathscr{L}(X)(X$-Banach space) which is continuous with respect to the topology inherited by $\mathscr{D}\left(R^{2}\right)$ from the space $\mathscr{B}_{0}\left(R^{2}\right)$ of $C^{\infty}$-functions on $R^{2}$ whose derivatives all vanish at infinity (cf. [3, p. 91] for the topology of $\mathscr{B}_{0}\left(R^{2}\right)$ ).

We say $T$ is an (unbounded) spectral distribution if and only if:

(a) $T_{\varphi \psi}=T_{\varphi} T_{\psi}, \forall \varphi, \psi \in \mathscr{D}\left(R^{2}\right)$.

(b) $T$ strongly vanishes to power 0 at infinity.

(c) $T$ is normalized; i.e., if $\varphi \in \mathscr{D}\left(R^{2}\right)$ is identically 1 near the origin in $R^{2}$, and if $\varphi_{n}(\lambda) \equiv \varphi(\lambda / n)$ for all $\lambda \in R^{2}$, then $T_{\varphi_{n}} x \rightarrow x$ as $n \rightarrow \infty$ for all $x \in X$.

We must show that property (c) is independent of the choice of the function $\varphi$. Let $\psi$ be another such function. Let $\eta \equiv \varphi-\psi, \eta_{n} \equiv \varphi_{n}-\psi_{n}$. We will show $T_{\eta_{n}} x \rightarrow 0$ as $n \rightarrow \infty$ for all $x \in X$. Now $\inf \left\{|\lambda|: \lambda \in \operatorname{supp} \eta_{n}\right\} \rightarrow \infty$ as $n \rightarrow \infty$, since $\eta$ vanishes near the origin in $R^{2}$. Furthermore, $\left\{\eta_{n}\right\}$ is a bounded subset of the space $\mathscr{B}_{0}\left(R^{2}\right)$, so that $\left\|T_{\eta_{n}}\right\|$ remains bounded as $n \rightarrow \infty$. Since $T$ strongly vanishes to power 0 at infinity, we conclude $T_{\eta_{n}} x \rightarrow 0$.

We remark that every spectral distribution in the sense of Foias, (cf. [5] or [2]) satisfies Definition 2. Furthermore, the mapping $T$ of equation (1) is a spectral distribution in the sense of Definition 2.

Definition 3. Let $A$ be a mapping of a subset $D_{A}$ of $X$ into $X . A$ is called an (unbounded) generalized scalar operator if and only if:

(a) There is a spectral distribution $T$ such that $x \in D_{A}$ if and only if $T$ strongly vanishes to power 1 at infinity for $x$ (and consequently $T_{\lambda \varphi_{n}} x$ converges, where $\varphi_{n}$ is as in Definition 2(c)) and

(b) $A x=\operatorname{limit} T_{\lambda \varphi_{n}} x$ for all $x \in D_{A}$.

We then say " $A$ admits the spectral distribution $T$ ". 
We must show that Definition 3 is independent of the choice of the function $\varphi$. With $\psi, \psi_{n}, \eta$, and $\eta_{n}$ as before, let $x \in X$. If $T$ strongly vanishes to power 1 at infinity for $x$, then $\left\|T_{\lambda \eta_{n}} x\right\| \rightarrow 0$ as $n \rightarrow \infty$, so $T_{\lambda \varphi_{n}} x$ and $T_{\lambda \psi_{n}} x$ have the same limit.

Every generalized scalar in the sense of Foias satisfies Definition 3. The operator of equation (2) is a generalized scalar in the sense of Definition 3. Furthermore, there are operators in Hilbert space which satisfy Definition 3 but are not normal, not even scalar in the sense of Dunford (cf. [5]).

\section{The domain of a generalized scalar operator.}

THEOREM 1. Let $A$ be a generalized scalar operator with spectral distribution $T$. Then

(a) The domain $D_{A}$ of $A$ is a linear manifold on which $A$ is a linear operator.

(b) $\bigcap_{n} D_{A^{n}}$ is dense in $X$; in fact, for all $x \in X$ and $\psi \in \mathscr{D}\left(R^{2}\right)$, we have $T_{\psi} x \in D_{A^{n}}$ and $A^{n} T_{\psi} x=T_{\lambda^{n} \psi} x$.

(c) $A T_{\psi} x=T_{\psi} A x$ for all $x \in D_{A}$ and $\psi \in \mathscr{D}\left(R^{2}\right)$.

(d) $D_{A^{n}}$ is the set of vectors for which $T$ vanishes to power $n$ at infinity.

Proof. (a) is clear.

(b) Since $T$ is normalized, the set $\left\{T_{\psi} x \mid x \in X, \psi \in \mathscr{D}\left(R^{2}\right)\right\}$ is dense. We prove $T_{\psi} x \in D_{A^{n}}$ and $A^{n} T_{\psi} x=T_{\lambda^{n} \psi} x$ by induction on $n$ : assuming the $n-1$ case, let $\eta \in \mathscr{D}\left(R^{2}\right)$, supp $\eta$ disjoint from supp $\psi$. Then $T_{\lambda \eta} A^{n-1} T_{\psi} x=$ $T_{\lambda \eta} T_{\lambda^{n-1} \psi} x=T_{\lambda^{n} \eta \psi} x=0$, so $T$ vanishes to power 1 at infinity for $A^{n-1} T_{\psi} x$. Thus $T_{\psi} x \in D_{A^{n}}$. And for $\left\{\varphi_{m}\right\}$ as in Definition 2(c),

$$
A^{n} T_{\psi} x=\lim _{m} T_{\lambda \varphi_{m}} T_{\lambda^{n-1} \psi} x=\lim _{m} T_{\lambda^{n} \varphi_{m} \psi} x=T_{\lambda^{n} \psi} x .
$$

(c) $T_{\psi} A x=\lim _{m} T_{\psi} T_{\lambda \varphi_{m}} x=\lim _{m} T_{\lambda \psi \varphi_{m}} x=T_{\lambda \psi} x=A T_{\psi} x$.

(d) Let $x \in D_{A^{n}}$. For $\eta \in \mathscr{D}\left(R^{2}\right)$, we have $T_{\lambda \eta} A^{n-1} x=T_{\lambda^{n} \eta} x$, so the vanishing of $T$ to power 1 at infinity for $A^{n-1} x$ implies $T$ vanishes to power $n$ at infinity for $x$.

Let $\mathscr{F}$ be a set of test functions on which $T$ is bounded. Let $\psi \in \mathscr{B}_{0}\left(R^{2}\right)$ be such that $\psi(\lambda)=1 / \lambda$ for $|\lambda|>1$. Then for $x \in X$ and $\eta \in \mathscr{F}$, we have

$$
\left\|T_{\psi \eta} x\right\|=\lim _{m}\left\|T_{\psi \eta \varphi_{m}} x\right\| \leqq\|x\| \cdot \sup _{\eta \in \mathscr{F}}\left\|T_{\eta}\right\| \cdot \sup _{m}\left\|T_{\psi \varphi_{m}}\right\| .
$$

But since $\left\{\psi \varphi_{m}\right\}$ is $\mathscr{B}_{0}\left(R^{2}\right)$-bounded, $\sup _{m}\left\|T_{\psi \varphi_{m}}\right\|$ is finite. Thus $T$ is bounded on $\{\psi \eta \mid \eta \in \mathscr{F}\}$. Together with the relation $T_{\lambda^{n-1} \eta}=T_{\lambda^{n} \psi \eta}$ for supp $\eta \cap S_{1}=\varnothing$, this implies that $T$ vanishes to power $n-1$ at infinity for $x$ if $T$ vanishes to power $n$ at infinity for $x$. The remaining half of statement (d) can thus be proved by induction on $n$ using the relation $T_{\lambda \eta} A^{n-1} x=$ $T_{\lambda^{n} \eta} x$ for $x \in D_{A^{n-1}}$. 
THEOREM 2. If $A$ is a generalized scalar operator, then $A$ is a closed operator.

Proof. Let $x_{m} \in D_{A}, x_{m} \underset{m}{ } x, A x_{m} \underset{m}{\rightarrow} y$. We first show that $T$ strongly vanishes to power 1 at infinity for $x$. Let $\psi$ run through a set of test functions for which $\left\|T_{\psi}\right\|$ remains bounded and $\inf \{|\lambda|: \lambda \in \operatorname{supp} \psi\} \rightarrow \infty$. Given $\varepsilon>0$, choose $m_{0}$ such that $m \geqq m_{0}$ implies $\left\|A x_{m}-y\right\| \leqq \varepsilon / 3 M$, where $M$ is a bound for $\left\|T_{\psi}\right\|$. Let $r_{m}$ be a positive number (for each $m$ ) chosen such that supp $\psi \cap S_{r_{m}}=\varnothing$ implies that $\left\|T_{\lambda \psi} x_{m}\right\| \leqq \varepsilon / 3$. Let $\psi$ be such that supp $\psi \cap$ $S_{r_{m_{0}}}=\varnothing$, and let $m>m_{0}$. Then

$$
\begin{aligned}
\left\|T_{\lambda \psi} x_{m}\right\| & \leqq\left\|T_{\lambda \psi} x_{m_{0}}\right\|+\left\|T_{\lambda \psi}\left(x_{m}-x_{m_{0}}\right)\right\| \\
& \leqq \varepsilon / 3+\left\|T_{\lambda \psi}\left(x_{m}-x_{m_{0}}\right)\right\| \\
& =\varepsilon / 3+\left\|T_{\psi} A\left(x_{m}-x_{m_{0}}\right)\right\| \\
& \leqq \varepsilon / 3+M\left\|A x_{m}-A x_{m_{0}}\right\| \leqq \varepsilon .
\end{aligned}
$$

Letting $m \rightarrow \infty$, we get $\left\|T_{\lambda \psi} x\right\| \leqq \varepsilon$ for supp $\psi \cap S_{r_{m_{0}}}=\varnothing$. Thus $T$ strongly vanishes to power 1 at infinity for $x$.

With $\varphi_{n}$ again as in Definition 2(c), Theorem 1(b) and (c) imply $T_{\lambda \varphi_{n}} x_{m}=T_{\varphi_{n}} A x_{m}$. Taking the limit as $m \rightarrow \infty$, we get $T_{\lambda \varphi_{n}} x=\operatorname{limit}_{m} T_{\varphi_{n}} A x_{m}=$ $T_{\varphi_{n}} y$. Taking the limit of this relation as $n \rightarrow \infty$, we get $\operatorname{limit}_{n} T_{\lambda \varphi_{n}} x=y$. Hence $x \in D_{A}$ and $A x=y$. This completes the proof of Theorem 2.

COROllaRY. If $A$ is a generalized scalar operator, then the spectrum $\sigma(A)$ of $A$ is a closed subset of $R^{2}$.

This follows from Theorem 2 and from Theorem 1 of $[9$, p. 211].

\section{The Spectral Mapping Theorem.}

THEOREM 3. Let $T$ be a spectral distribution, and $\psi \in \mathscr{D}\left(R^{2}\right)$. Then (a) $T_{\psi}$ is a (bounded) generalized scalar operator; and

(b) $\sigma\left(T_{\psi}\right) \subset \operatorname{cl}(\psi(\operatorname{supp} T))$.

Proof of (a). For $\eta \in \mathscr{D}\left(R^{2}\right)$, define a function $\tilde{\eta}$ by the formula

$$
\tilde{\eta}(\lambda) \equiv \eta(\psi(\lambda))-\eta(0) \text {. }
$$

Then $\tilde{\eta} \in \mathscr{D}\left(R^{2}\right)$. Define a mapping $S: \mathscr{D}\left(R^{2}\right) \rightarrow \mathscr{L}(X)$ by

$$
S_{\eta} \equiv T_{\tilde{\eta}}+\eta(0) I \text {. }
$$

Then clearly $S$ is linear, and continuous with respect to the $\mathscr{B}_{0}\left(R^{2}\right)$ topology of $\mathscr{D}\left(R^{2}\right)$. If $\varphi_{n}$ is as in Definition 2(c), if $n$ is large enough so that $\varphi_{n} \equiv 1$ near supp $\psi$, and if $\eta \in \mathscr{D}\left(R^{2}\right)$, then $\tilde{\eta} \varphi_{n}=\tilde{\eta}$, so that for every $x \in X$ we have

$$
S_{\eta} x=T_{\tilde{\eta} \varphi_{n}} x+\eta(0) I x=\underset{n}{\operatorname{limit}}\left(T_{\tilde{r} \varphi_{n}}+\eta(0) T_{\varphi_{n}}\right) x=\operatorname{limit}_{n} T_{\left(\eta_{\circ} \psi\right) \varphi_{n}} x .
$$


Now if both $m$ and $n$ are large enough so that $\varphi_{n}$ and $\varphi_{m}$ are identically 1 near supp $\psi$, then for $\eta_{1}, \eta_{2} \in \mathscr{D}\left(R^{2}\right)$ we have

$$
T_{\left[\left(\eta_{1} \eta_{2}\right) \circ \psi\right] \varphi_{n} \varphi_{m}} x=T_{\left(\eta_{1} \circ \psi\right) \varphi_{n}} T_{\left(\eta_{2} \circ \psi\right) \varphi_{m}} x .
$$

Taking the limit as $m \rightarrow \infty$, we get

$$
T_{\left[\left(\eta_{1} \eta_{2}\right) \circ \psi\right] \varphi_{n}} x=T_{\left(\eta_{1} \circ \psi\right) \varphi_{n}} S_{\eta_{2}} x .
$$

Taking the limit as $n \rightarrow \infty$, we get $S_{\eta_{1} \eta_{2}} x=S_{\eta_{1}} S_{\eta_{2}} x$.

We will show that $\operatorname{supp} S \subset \operatorname{cl}(\psi(\operatorname{supp} T))$, a compact set. If $\eta \in \mathscr{D}\left(R^{2}\right)$, $\operatorname{supp} \eta \cap \operatorname{cl}(\psi(\operatorname{supp} T))=\varnothing$, then $\eta \circ \psi=0$ near $\operatorname{supp} T$, so $S_{\eta} x=$ $\operatorname{limit}_{n} T_{(\eta, \psi) \phi_{n}} x=0$ for all $x \in X$. Since $S$ has compact support, $S_{1} x=$ $\operatorname{limit}_{n} T_{(1 \circ \psi) \varphi_{n}} x=x$ for all $x \in X$. Thus $S$ is a spectral distribution in the sense of Foiaș. Furthermore, $S_{\lambda} x=\operatorname{limit}_{n} T_{\left(\lambda_{0} \psi\right) \varphi_{n}} x=T_{\psi} x$ for all $x \in X$. Thus $T_{\psi}$ is a generalized scalar in the sense of Foias with spectral distribution $S$.

Proof of (b). By Foias' Spectral Mapping Theorem (Proposition 1(ii) of [2]), we have $\sigma\left(T_{\psi}\right)=\operatorname{supp} S \subset \operatorname{cl}(\psi(\operatorname{supp} T))$.

TheOREM 4 (SPECTRAL MAPPING THEOREM). If $A$ is a generalized scalar operator with spectral distribution $T$, then $\sigma(A)=\operatorname{supp} T$.

We prove this theorem in two steps:

(a) $\sigma(A) \subset \operatorname{supp} T$.

(b) If the resolvent set $\rho(A)$ of $A$ is nonempty, then $\sigma(A)=\operatorname{supp} T$.

Proof of (a). Assume $\lambda_{0} \notin \operatorname{supp} T$. Let $\varphi_{n}$ be as in Definition 2(c). Then there is a function $\chi$ from the space $\mathscr{B}\left(R^{2}\right)$ of $C^{\infty}$ functions with bounded derivatives of all orders such that $\chi(\lambda)=\left(\lambda_{0}-\lambda\right)^{-1}$ near supp $T$. Hence $\chi\left(\lambda_{0}-\lambda\right) \varphi_{n}=\varphi_{n}$ near supp $T$, so that for any $x \in D_{A}$ we have

$$
T_{\chi \varphi_{n}}\left(\lambda_{0} I-A\right) x=\left(\lambda_{0} I-A\right) T_{\chi \varphi_{n}} x=T_{\chi\left(\lambda_{0}-\lambda\right) \varphi_{n}} x=T_{\varphi_{n}} x \underset{n}{\longrightarrow} x .
$$

This implies that $\lambda_{0} I-A$ is one-to-one, its range is dense (since $D_{A}$ is dense), and $T_{\chi \varphi_{n}} y \vec{n}_{n}\left(\lambda_{0} I-A\right)^{-1} y$ for all $y$ in the range of $\lambda_{0} I-A$. Furthermore, $\left\{\chi \varphi_{n}\right\}$ is a bounded subset of $\mathscr{B}_{0}\left(R^{2}\right)$, so $\left\|T_{\chi \varphi_{n}}\right\|$ are bounded. Thus $\left(\lambda_{0} I-A\right)^{-1}$ is a bounded operator and $\lambda_{0} \notin \sigma(A)$. This proves $\sigma(A) \subset \operatorname{supp} T$.

Proof of (b). We show supp $T \subset \sigma(A)$. Let $\varphi \in \mathscr{D}\left(R^{2}\right)$, supp $\varphi \cap \sigma(A)=$ $\varnothing$. Define a map $\mathscr{R}:\left(R^{2} \backslash \operatorname{supp} \varphi\right) \rightarrow \mathscr{D}\left(R^{2}\right)$ by

$$
\mathscr{R}_{\zeta}=\varphi(\lambda)(\zeta-\lambda)^{-1}, \quad \forall \lambda \in R^{2},
$$

for each $\zeta \notin \operatorname{supp} \varphi . \mathscr{R}$ is an analytic function from $\left(R^{2} \backslash \operatorname{supp} \varphi\right)$ into $\mathscr{D}\left(R^{2}\right)$. Therefore the mapping $f$ from $\left(R^{2} \backslash \operatorname{supp} \varphi\right)$ into $\mathscr{L}(X)$ defined by $f_{\zeta}=T \mathscr{R}_{\zeta}$ is an analytic function. Furthermore the function $g: \rho(A) \rightarrow \mathscr{L}(X)$ defined by $g_{\zeta}=(\zeta I-A)^{-1} T_{\varphi}$ is also analytic (cf. [9, p. 211]). If $\zeta \in D_{f} \cap D_{g}=$ $R^{2} \backslash(\sigma(A) \cup \operatorname{supp} \varphi)$ then

$$
(\zeta I-A) f_{\zeta}=(\zeta I-A) T_{\varphi(\zeta-\lambda)^{-1}}=T_{\zeta \varphi(\zeta-\lambda)^{-1}}-T_{\lambda \varphi(\zeta-\lambda)^{-1}}=T_{\varphi},
$$


so that $f_{\zeta}=g_{\zeta}$. Hence there is an entire analytic function $F: R^{2} \rightarrow \mathscr{L}(X)$ agreeing with $f$ and $g$ where each is defined. Now as $\left|\zeta_{n}\right| \rightarrow \infty$, the functions $\psi_{n} \equiv \varphi \cdot\left(\zeta_{n}-\lambda\right)^{-1}$ converge to 0 in $\mathscr{D}\left(R^{2}\right)$. Thus $T_{\psi_{n}}=F_{\zeta_{n}} \rightarrow 0$ as $n \rightarrow \infty$. By Liouville's theorem, we conclude that $F \equiv 0$ in $R^{2}$. If $\zeta \in \rho(A) \neq \varnothing$, then $0=F_{\zeta}=g_{\zeta}=(\zeta I-A)^{-1} T_{\varphi}$ so that $T_{\varphi}=0$, and we have supp $T \subset \sigma(A)$.

\section{REFERENCES}

1. I. Colojoară and C. Foiaş, Quasi-nilpotent equivalence of not necessarily commuting operators, J. Math. Mech. 15 (1966), 521-540. MR 33 \#570.

2. C. Foias, Une application des distributions vectorielles à la théorie spectrale, Bull. Sci. Math. (2) 84 (1960), 147-158. MR 23 \#A522.

3. J. Horváth, Topological vector spaces and distributions, Vol. I, Addison-Wesley, Reading, Mass., 1966. MR 34 \#4863.

4. B. Kritt, Spectral decomposition of positive and positive-definite distributions of operators, Bull. Acad. Polon. Sci. Sér. Sci. Math. Astronom. Phys. 16 (1968), 865-870. MR 39 \#3307.

5. — Generalized pseudo-hermitian operators, Proc. Amer. Math. Soc. 30 (1971), 343-348.

6. F. Maeda, Generalized spectral operators on locally convex spaces, Pacific J. Math. 13 (1963), 177-192. MR 33 \#1732.

7. P. Masani, Orthogonally scattered measures, Advances in Math. 2 (1968), 61-117. MR 37 \#4231.

8. H. G. Tillman, Vector-valued distributions and the spectral theorem for selfadjoint operators in Hilbert space, Bull. Amer. Math. Soc. 69 (1963), 67-71. MR 26 \#5423.

9. K. Yosida, Functional analysis, Die Grundlehren der math. Wissenschaften, Band 123, Academic Press, New York; Springer-Verlag, Berlin, 1965. MR 31 \#5054.

10. S. Kantorovitz, Classification of operators by means of the operational calculus, Bull. Amer. Math. Soc. 70 (1964), 316-320. MR 30 \#460.

Department of Mathematics, University of Baltimore, Baltimore, Maryland 21201 